of Chinese children to receive input from health professionals. For example, some Chinese parents may not feel confident in bringing a young person with a suspected mental health difficulty to see a general practitioner, and Chinese carers may find it difficult to understand concerns regarding their child's emotional well-being as communicated to them by professionals such as teachers. Furthermore, systemic (e.g. family therapy) or parenting-based work may be difficult, especially in the absence of an independent (non-family) interpreter.

In addition to language issues, cultural factors may also shape help-seeking behaviour. Although present across cultures, the problem of stigma remains prominent among the Chinese population. For example, a preliminary assessment of the mental health needs of Chinese young people in Birmingham revealed that the majority of them perceived mental illness as being 'crazy' and 'associated with violence' (Fung, 2005). Such cultural and individual attitudes could serve to prevent or at least delay young people and their families from engaging with mental health services.

Research is urgently needed in order to develop an understanding of the mental health needs of Chinese children in the UK. This should feed into developing programmes of public education and more culturally acceptable services in order to increase the Chinese community's access to timely help for young people. Without this, UK health services will find it difficult to meet agreed racial equality goals.

W. Cheng ${ }^{1}$ and P. A. Tiffin ${ }^{2}$

${ }^{1}$ Specialty Registrar in Child and Adolescent Psychiatry, Northumberland, Tyne and Wear NHS Trust, email wilson.cheng@ntw.nhs.uk; ${ }^{2}$ Clinical Senior Lecturer and Honorary Consultant in the Psychiatry of Adolescence, Durham University and Tees, Esk and Wear Valleys NHS Foundation Trust

Cowan, C. (2001) The mental health of Chinese people in Britain: an update on current literature. Journal of Mental Health, 10, 501-511.

Fung, R. (2005) Stigma of mental illness among Chinese people. Psychiatric Bulletin, 29, 193

Goodman, A., Patel, V. \& Leon, D. A. (2008) Child mental health differences amongst ethnic groups in Britain: a systematic review. BMC Public Health, 8, 258.

\section{Proportionality of legal discrimination}

SHe= The article by Zigmond (2009) made for SI interesting reading. Mental health law is about balancing the need to detain people in order to protect them or other people from harm and the need to respect people's human rights and autonomy. In the UK, there was much concern during the development of recent mental health legislation, in particular the Mental Capacity Act 2005, that the government had got this balance wrong. Many of these concerns have been addressed in the updated Code of Practice to the 1983 Mental Health Act, which is an essential guide to practising under the Act (Department of Health, 2008). There is no legal duty to comply with the Code, but professionals must have regard to it and record the reason for any departure from the guidance (which can be subject to legal challenge).
Safeguards regarding deprivation of liberty, which address the 'Bournewood gap' concerning the detention of compliant incapacitous individuals, in the Mental Capacity Act have been one of the highlights of the changes introduced (Hall \& Ali, 2009). The mental health legislation in England and Wales is based on risk. One of the arguments for having risk as the main focus is the fact that mental illness leads to loss of insight, which makes it impossible for the sufferer to make an informed decision. The proponents of the other view argue that having a different criterion for compulsory treatment (risk rather than capacity) for mental illness results in further discrimination against people who are mentally ill and can only help to enhance stigma.

The European Court of Human Rights has had some impact on the Mental Health Act 1983 and its interpretation; it has not, however, set a high standard for modern mental health services. Some judgements may strike present-day clinicians not so much as protecting patients' rights but as permitting undesirable practices. This is perhaps not surprising when it is considered that the European Convention on Human Rights, signed in 1950, harbours old prejudices against those with mental illness (Bindman et al, 2003). These are apparent in the language of Article 5, which groups persons of 'unsound mind' with 'vagrants' and 'drug addicts' as being exempted from the protections afforded to others. In incorporating the European Convention on Human Rights, the UK Human Rights Act 1998 perpetuates rather than challenges the lesser regard for the autonomy of patients with mental illness than of other medical patients, which is at the heart of conventional mental health legislation (Szmukler \& Holloway, 2000). If the courts do begin to scrutinise the proportionality of clinical decisions - a function currently carried out only haphazardly by mental health review tribunals (Perkins, 2000) - the impact could be considerable. Many of the cases involving the European Convention on Human Rights to date have concerned patients in maximum security settings or with significant forensic histories, and it is not surprising that compulsory treatment is often found to be justified or the infringement of rights to be proportionate. However, a decision, for example, to compel a 'revolving door' patient without a history of offending to accept community treatment might be judged to be disproportionate if founded on weak scientific evidence of risk or benefit.

Dr Partha Gangopadhyay Clinical Teaching Fellow and Honorary Specialty Registrar in Forensic Psychiatry, University of Aberdeen, UK, email p.gangopadhyay@abdn.ac.uk

Bindman, J., Maingay, S. \& Szmukler, G. (2003) The Human Rights Act and mental health legislation. British Journal of Psychiatry, 182, 91-94.

Department of Health (2008) Code of Practice, Mental Health Act 1983. TSO (The Stationery Office).

Hall, I. \& Ali, A. (2009) Changes to the Mental Health and Mental Capacity Acts: implications for patients and professionals. Psychiatric Bulletin, 33, 226-230.

Perkins, E. (2000) Decision-making in mental health review tribunals. In Shaping the New Mental Health Act: Key Messages from the Department of Health Research Programme, pp. 29-32. Department of Health.

Szmukler, G. \& Holloway, F. (2000) Reform of the Mental Health Act: health or safety? British Journal of Psychiatry, 177, 196-200.

Zigmond, T. (2009) Mental illness and legal discrimination. International Psychiatry, 6, 79-80. 